\title{
On Instantaneous Amplitude and Phase of Signals
}

\author{
Bernard Picinbono, Fellow, IEEE
}

\begin{abstract}
In many questions of signal processing, it is important to use the concepts of instantaneous amplitude or phase of signals. This is especially the case in communication systems with amplitude or frequency modulation. These concepts are often introduced empirically. However, it is well known that the correct approach for this purpose is to use the concept of analytic signal. Starting from this point, we show some examples of contradictions appearing when using other definitions of instantaneous amplitude or frequency that are commonly admitted. This introduces the problem of characterizing pure amplitudemodulated or pure phase-modulated signals. It is especially shown that whereas amplitude modulated signals can be characterized by spectral considerations, this is no longer the case for phasemodulated signals. Furthermore, signals with constant amplitude have very specific properties, which are analyzed in detail. Some consequences and extensions to random signals are finally discussed.
\end{abstract}

\section{INTRODUCTION}

$\mathbf{I}$ NSTANTANEOUS amplitude and phase are basic concepts in all the questions dealing with modulation of signals appearing especially in communications or information processing. Let us remember that a purely monochromatic signal such as $a \cos (\omega t+\phi)$ cannot transmit any information. For this purpose, a modulation is required, and one of the simplest possible to introduce is amplitude modulation. Let $m(t)$ be a positive function corresponding to the information to be transmitted. By multiplying the carrier frequency signal $\cos \left(\omega_{0} t\right)$ by $m(t)$, we obtain the signal

$$
x(t)=m(t) \cos \left(\omega_{0} t\right)
$$

and it is commonly admitted that $m(t)$ is the instantaneous amplitude of the signal $x(t)$. This appears in many textbooks, especially in [1, p. 237]. On the other hand, the need for phase or frequency modulation requires the definition of the instantaneous phase. By a reasoning similar to the previous one concerning the amplitude, it is commonly admitted that the signal

$$
x(t)=a \cos [\phi(t)]
$$

has a constant amplitude $a$ and an instantaneous phase $\phi(t)$. Furthermore, the instantaneous angular frequency is given by the derivative of $\phi(t)$. This is a generalization of the procedure applied in the case where the phase is linear in time or in the form $\phi(t)=\omega_{0} t+\phi$, giving the frequency $\omega_{0}$. These definitions also appear in many textbooks or papers.

Manuscript received December 30, 1995; revised October 11, 1996. The associate editor coordinating the review of this paper and approving it for publication was Dr. Bruce W. Suter.

The author is with the Laboratoire des Signaux et Systèmes, Supélec, Gif sur Yvette, France (e-mail: picinbono@1ss.supelec.fr).

Publisher Item Identifier S 1053-587X(97)01862-X
For example, the following is written on [2, p. 645]: "The argument $\theta(t)$ in any signal having the form $\cos [\theta(t)]$ is called the instantaneous phase, and $(1 / 2 \pi) d[\theta(t)] / d t$ is called the instantaneous frequency." The same definition appears, for example, in [3, p. 480], [4, p. 260], and [5, p. 144].

Even if the previous definitions appear quite natural and are widely used in practical applications dealing with signal modulation, we immediately note that they cannot be satisfactory. In order to make this point clear, let us discuss a very simple example. Suppose that the function $m(t)$ appearing in (1) is bounded or satisfies $0 \leq m(t) \leq a$. As a result, we have $|(1 / a) x(t)| \leq 1$. It is then possible to introduce a unique function $\phi(t)$ satisfying

$$
0 \leq \phi(t) \leq \pi ; \quad \cos [\phi(t)]=(1 / a) x(t) .
$$

By using this well-defined function, we obtain

$$
x(t)=m(t) \cos \left[\omega_{0} t\right]=a \cos [\phi(t)] .
$$

This shows that the signal $x(t)$ can be considered frequency as well as amplitude modulated. In other words, its instantaneous amplitude is $m(t)$ as well as $a$, and moreover, its instantaneous phase is either $\omega_{0} t$ or $\phi(t)$.

More generally, starting from a given signal $x(t)$, it is possible to introduce an infinite number of pairs $[a(t), \phi(t)]$ such that

$$
x(t)=a(t) \cos [\phi(t)] .
$$

This leads to the conclusion that the definitions given previously, even if they are widely used, are incoherent because they do not associate with a given real signal $x(t)$ a well-defined pair of functions that are the instantaneous amplitude and phase of $x(t)$. Therefore, these definitions must be reformulated in such a way that any given signal $x(t)$ corresponds to one well-defined pair $[a(t), \phi(t)]$, allowing us to write $x(t)$, as in (5).

In reality, the solution of this problem is well known and explicitly introduced in [6, p. 50]. A recent review paper on this question [7] also gives a good introduction on the discussion leading to the standard definition of the quantities studied hereafter. Moreover, this paper contains a list of references corresponding to the history of this problem.

The classical definition of the instantaneous amplitude $a(t)$ and phase $\phi(t)$ of a real signal $x(t)$ is recalled in the next section. This definition introduces the concept of a canonical pair $[a(t), \phi(t)]$, and it is therefore interesting to find conditions ensuring that a given pair of functions $a(t)$ and $\phi(t)$ is canonical. When the instantaneous phase is linear, or when the signal looks like (1), it is possible to characterize a canonical pair only from the spectral properties of the signal. This 
becomes much more difficult when the instantaneous phase is no longer linear, and this especially appears for signals with constant amplitude, which are called phase signals, which are at the foundation of phase- or frequency-modulation systems. The properties of such signals are analyzed in the following section, and from them, we can deduce various properties of canonical pairs. The practical consequences of these results and their extension for random signals are finally investigated.

\section{DEFINITIONS AND CANONICAL PAIRS}

\section{A. Definitions}

Let us recall the classical way to define without ambiguity the instantaneous amplitude and phase of a real signal $x(t)$. The problem is to write $x(t)$ as in (5) but by using a pair of functions $[a(t), \phi(t)]$ that is in a one-to-one correspondence with $x(t)$. For this purpose, we associate with $x(t)$ its analytic signal (AS) $z(t)$ (see [6, p. 48]). It is obtained from $x(t)$ by filtering it using a filter with the frequency response $H(\nu)$ equal to 2 for $\nu>0$ and to 0 for $\nu<0$. Conversely, it is obvious that $x(t)=\operatorname{Re}[z(t)]$, where $\operatorname{Re}$ means the real part. Therefore, if $x(t)$ is real, there is indeed a one-to-one correspondence between $x(t)$ and $z(t)$. On the other hand, a complex function is an AS if its Fourier transform is zero for negative frequencies. It is clear that this function is the AS of its real part.

As $z(t)$ cannot be a real function because its Fourier transform $Z(\nu)$ is zero for $\nu<0$, it can be written as

$$
z(t)=a(t) \exp [j \phi(t)]
$$

where the phase $\phi(t)$ is defined modulo $2 \pi$, and $a(t)$ is nonnegative. As a conclusion, using the AS makes it possible to associate with any real signal a unique pair $[a(t), \phi(t)]$ called in the following the canonical pair associated with $x(t)$.

Definition: Let $x(t)$ be a real signal and $[a(t), \phi(t)]$ the canonical pair associated with it. The function $a(t)$ appearing in this pair is the instantaneous amplitude of $x(t)$, and $\phi(t)$ is its instantaneous phase. The instantaneous frequency is the derivative with respect to time of $\phi(t)$.

The introduction of the AS is not at all recent, and among the principal papers in this field, we can note [8]-[12]. There are some questions concerning the physical meaning of the AS, and some of them are mentioned and discussed in [7]. Furthermore, it is shown in [13] and [14] that starting from some a priori physical assumptions, the only possible definition of the instantaneous amplitude and phase is the one given just above. However, it is worth pointing out that other physical conditions lead to another definitions that are not discussed below [15].

Once the definition is given, the question that immediately follows is to characterize a canonical pair or to show what the conditions are on $a(t)$ and $\phi(t)$ in order to ensure that (6) is an AS, which means that its Fourier transform is zero for negative frequencies. This is essential when verifying whether or not the classical definitions given above are correct. In fact, there is no a priori reason for $\left[m(t), \omega_{0} t\right]$ appearing in (1) or $[a, \phi(t)]$ appearing in (2) to be canonical.

\section{B. Spectral Characterization of a Canonical Pair}

Consider first a signal of the form (1). It corresponds to an amplitude modulation of a pure monochromatic signal with the carrier frequency $\omega_{0}$. The nonnegative function $m(t)$ is the instantaneous amplitude of $x(t)$ if and only if $m(t) \exp \left(j \omega_{0} t\right)$ is an AS.

Let $M(\nu)$ be the Fourier transform (FT) of $m(t)$. As $m(t)$ is real, $M(\nu)=M^{*}(-\nu)$. The FT of $m(t) \exp \left(j \omega_{0} t\right)$ is, of course, $M\left(\nu-\nu_{0}\right)$, with $\nu_{0}=\omega / 2 \pi$. As a result, we obtain that $M\left(\nu-\nu_{0}\right)=0$ for $\nu$ negative or that $m(t)$ is the instantaneous amplitude of $x(t)$ given by (1) if and only if $M(\nu)$ is zero for $|\nu|>\nu_{0}$. Physically, this means that $m(t)$ is a low-frequency bandlimited signal. In all that follows, we call such a signal a low-frequency $\left(\nu_{0}\right)$ signal. Similarly, a high-frequency $(B)$ signal is characterized by the fact that its FT vanishes for $|\nu|<B$.

This discussion shows that it is possible to characterize a canonical pair such as $\left[a(t), \omega_{0} t\right]$ uniquely by a spectral condition on $a(t)$. Starting from this example of amplitude modulation, it was tempting to try to use spectral methods for the characterization of more general pairs of functions $[a(t), \phi(t)]$. Unfortunately, we shall see that the task becomes immediately impossible.

Saying that $a(t) \exp [j \phi(t)]$ is an AS is equivalent to saying that the Hilbert transform of $a(t) \cos [\phi(t)]$ is equal to $a(t) \sin [\phi(t)]$ (see [6, p. 49]). It is therefore appropriate to make use of the so-called Bedrosian theorem [16] dealing with the Hilbert transform of a product of two real functions $x_{1}(t)$ and $x_{2}(t)$. A very simple derivation of this theorem and some extensions can be found in [17]. The main result is as follows: Let $X_{1}(\nu)$ and $X_{2}(\nu)$ be the FT's of $x_{1}(t)$ and $x_{2}(t)$, respectively. If $X_{1}(\nu)=0$ for $\nu>B$ and $X_{2}(\nu)=0$ for $\nu<B$, then

$$
H\left[x_{1}(t) x_{2}(t)\right]=x_{1}(t) H\left[x_{2}(t)\right]
$$

where $H[\cdot]$ means the Hilbert transform. A direct application of this result shows that if $a(t)$ is a low-frequency signal $(B)$, and $\cos [\phi(t)]$ a high-frequency signal $(B)$, or if their spectra do not overlap, then

$$
H\{a(t) \cos [\phi(t)]\}=a(t) H\{\cos [\phi(t)]\} .
$$

However, this does not at all imply that

$$
H\{\cos [\phi(t)]\}=\sin [\phi(t)]
$$

as stated by many authors and even recently in [7]. If (9) were true, it would also be possible to characterize a canonical pair only by spectral considerations, as for amplitude modulation. Furthermore, as the constant signal has an FT limited to the frequency zero, (9) would be true for any signal $\cos [\phi(t)]$ without a low-frequency component. This result would be especially attractive, suppressing all the questions discussed in the introduction, when presenting some comments on (2).

Let us now show that (9) has no reason to be true when only spectral properties of $\cos [\phi(t)]$ are introduced. For this purpose, we shall propose an elementary counterexample of this property. It is obvious that (9) implies that

$$
\cos ^{2}[\phi(t)]+(H\{\cos [\phi(t)]\})^{2}=1 .
$$


Let $x(t)$ be defined by

$$
x(t)=\operatorname{sinc}(2 B t) \cos \left(\omega_{0} t\right)
$$

where $\operatorname{sinc} x=\sin (\pi x) /(\pi x)$, and $\omega_{0}>2 \pi B$. As $|x(t)| \leq 1$, it is possible to introduce a phase $\phi(t)$ uniquely defined if $0 \leq \phi(t) \leq \pi$ and such that $x(t)=\cos [\phi(t)]$. As the FT of $\operatorname{sinc}(2 B t)$ is zero for $|\nu|>B$, the FT of $x(t)$ does not contain low-frequency components because of the assumption on $\omega_{0}$. Applying (9) then gives $H\{x(t)\}=\sin [\phi(t)]$, and as a consequence, $x^{2}(t)+H^{2}\{x(t)\}=1$. However, it is obvious that this equality is not correct. In fact, by applying the Bedrosian theorem, we obtain

$$
H\{x(t)\}=\operatorname{sinc}(2 B t) \sin \left(\omega_{0} t\right)
$$

and $x^{2}(t)+H^{2}\{x(t)\}=\operatorname{sinc}^{2}(2 B t)$.

This shows that contrary to a common well-established idea, it is not possible to justify (9) by introducing only spectral considerations. This point will become much clearer in the next section. In fact, (9) implies that

$$
z(t)=\exp [j \phi(t)]
$$

is an AS or that the pair $[1, \phi(t)]$ is canonical. We shall now see that this requires very specific properties of the structure of the phase $\phi(t)$.

\section{Phase Signals}

\section{A. General Structure}

Phase signals are real signals with constant instantaneous amplitude. They can be expressed as (2) but with the condition that $\exp [j \phi(t)]$ is an AS. As a consequence, (9) is satisfied. For such signals, all the information is contained in the instantaneous phase (or frequency), and phase signals are then the basic elements of phase or frequency modulation.

The condition that $\exp [j \phi(t)]$ is an AS requires very specific properties on the phase $\phi(t)$. These properties have been analyzed in the framework of coherence problems in optics [18] but, more precisely, in the framework of the study of analytic functions, and especially in [19, ch. 7]. We present here the results that are the most important for our arguments without the proofs, which are out of the scope of this discussion and can be found in [19].

The most general structure of the AS of a nonsingular phase signal is

$$
z(t)=b(t) \exp \left[j\left(\omega_{0} t+\theta\right)\right]
$$

where $\theta$ is arbitrary, $\omega_{0}$ is nonnegative, and $b(t)$ is a Blaschke function defined by

$$
b(t)=\prod_{k=1}^{N} \frac{t-z_{k}}{t-z_{k}^{*}}, \quad z_{k} \in P_{+}
$$

where $P_{+}$is the half plane of the complex plane defined by $\operatorname{Im}(z)>0$. The quantity $\omega_{0}$ is the carrier frequency and can be equal to zero. The expression nonsingular means that the instantaneous phase of $z(t)$ remains finite for finite values of $t$. When the number $N$ of factors in the product is not finite, there are other constraints on the complex numbers $z_{k}$ due to convergence problems. Here, we avoid these questions by assuming that $N$ is finite. In this case, the interpretation of (15) is very simple. In order to be an AS, the function $b(t)$, for complex values of $t$, must have all its poles in the half plane $\operatorname{Im}(z)<0$. In order to have a modulus equal to one, each pole must be associated with a corresponding zero symmetric of this pole with respect to the real axis. This procedure is well known in filter theory: Stable phase filters have the same number of poles and zeros, and these zeros are symmetric to the poles with respect to the imaginary axis. The stability and causality conditions imply that all the poles are in the left half plane of the complex plane.

It is obvious that $|b(t)|=1$, which implies that $|z(t)|=1$. Let us now explain why $z(t)$ is an AS. For this, we must analyze the structure of the FT $B(\nu)$ of $b(t)$. As $N$ is finite, $b(t)$ is a rational function in $t$. If all the $z_{k}$ s are distinct, we can write

$$
b(t)=1+\sum_{k=1}^{N} \frac{c_{k}}{t-z_{k}^{*}}
$$

where

$$
c_{k}=\lim _{t \rightarrow z_{k}^{*}}\left(t-z_{k}^{*}\right) b(t) .
$$

As a consequence, we have

$$
B(\nu)=\delta(\nu)+\sum_{k=1}^{N} C_{k}(\nu)
$$

where $C_{k}(\nu)$ is the FT of $c_{k}\left(t-z_{k}^{*}\right)^{-1}$. Because of the localization of $z_{k}^{*}$ in the complex plane, we deduce that $C_{k}(\nu)=0$ for $\nu<0$, which implies that $B(\nu)=0$ for $\nu<0$ and ensures that $b(t)$ is an AS. Finally, as $\omega_{0}>0$, $z(t)$ also is an AS. The reasoning can be extended without difficulty when some poles $z_{k}$ are no longer distinct.

The phase of $b(t)$ is, of course

$$
\phi_{b}(t)=\operatorname{Arg}[b(t)], \bmod (2 \pi)
$$

and, as a result, we can say that any phase signal can be written as (2), where $\phi(t)$ must have the form

$$
\phi(t)=\theta+\omega_{0} t+\phi_{b}(t), \bmod (2 \pi) .
$$

In practice, the continuity of the phase leads to suppress the term $\bmod (2 \pi)$, and this convention is adopted in all that follows.

This most general phase is defined by $N$ complex parameters $z_{k}$ and two real parameters $\omega_{0}$ and $\theta$. Furthermore, it is obvious that the phase $\phi_{b}(t)$ is the sum of $N$ phases of the factors appearing in the product $(15)$. Let $b_{k}(t)$ be equal to $\left(t-z_{k}\right)\left(t-z_{k}^{*}\right)^{-1}$, and let $\phi_{k}(t)$ be its phase. This gives

$$
\phi(t)=\theta+\omega_{0} t+\sum_{k=1}^{N} \phi_{k}(t) .
$$

By introducing the real and imaginary parts of $z_{k}$ or $z_{k}=$ $a_{k}+j b_{k}$, one obtains

$$
\phi_{k}(t)=2 \operatorname{Arctg} \frac{b_{k}}{a_{k}-t}, \quad-\pi / 2 \leq \phi_{k}(t) \leq \pi / 2 .
$$


At this step, we come back to the problem discussed in the introduction. The signal (2) is phase modulated only if its phase takes the form (20), and as this is not in general the case, its amplitude is not constant, and it must be expressed as in (5).

\section{B. Properties of Phase Signals}

Having the most general structure of phase signals, we will now present some of their properties, which allows a better understanding of their structure.

Property 3.1: A phase signal contains only two spectral lines corresponding to its carrier frequency. This is a direct consequence of the structure of the Blaschke function appearing in (15). Its FT $B(\nu)$ is given by (18), which can be written as

$$
B(\nu)=\delta(\nu)+B_{c}(\nu)
$$

The function $B_{c}(\nu)$ describes the continuous part of the FT of $B(\nu)$. It is a sum of $N$ components $C_{k}(\nu)$ that are bounded and equal to zero for $\nu<0$. This implies that $B_{c}(\nu)$ is also bounded and equal to zero for $\nu<0$, and thus, $B(\nu)$ exhibits only one Dirac component, or a spectral line, at the frequency zero. Because of the exponential term in (14), the FT of $z(t)$ is $Z(\nu)=e^{j \theta} B\left(\nu-\nu_{0}\right)$, and this means that there is only one spectral line at the carrier frequency $\nu_{0}=\omega_{0} / 2 \pi$. By using the Hermitian symmetry, we deduce that $x(t)$ has only two spectral lines at the frequencies $\pm \nu_{0}$.

This property can be used in a reciprocal way, indicating that all the signals containing more that one spectral line in the range of positive frequencies cannot be phase signals and, therefore, exhibit a nonconstant instantaneous amplitude.

Property 3.2: A phase signal with a nonzero carrier frequency is a high-frequency $\left(\nu_{0}\right)$ signal. This is a direct consequence of the form of the FT $Z(\nu)$ analyzed just above. As $B(\nu)=0$ for $\nu<0, Z(\nu)=0$ for $\nu<\nu_{0}$, and $X(\nu)=0$ for $|\nu|<\nu_{0}$.

The converse is, of course, not true. There is no reason for a high-frequency signal to be a phase signal because this frequency condition does not imply the structure (14). A simple counterexample appears with a high-frequency signal containing more than two spectral lines and, thus, does not satisfy Property 3.1.

Property 3.3: The FT of the AS of a phase signal is zero for all the frequencies smaller than the carrier frequency $\nu_{0}$ where the spectral line is located. This is a direct consequence of (23) and of the fact that the FT $Z(\nu)$ of $z(t)$ is proportional to $B\left(\nu-\nu_{0}\right)$

Property 3.4: A phase signal cannot be a low-frequency $(B)$ signal except when it is monochromatic. The monochromatic case appears when $b(t)=1$, and $z(t)$ is therefore $\cos \left(\omega_{0} t+\theta\right)$, that is, of course, a low-frequency signal. Except in this case, the property means that it is impossible to find a frequency $B$ such that $Z(\nu)=0$ for $\nu>B$. This property can be shown by two procedures. In the first, we simply note that the functions $C_{k}(\nu)$ of (18) are exponential functions for $\nu>0$ when the poles are distinct. However, it is well known that a sum of a finite number of exponential functions cannot be zero for all the frequencies satisfying $\nu>B$.

It is also possible to show this property by contradiction. Suppose then that there exists a frequency $B$ such that $Z(\nu)=$ 0 for $\nu>B$. It results from this assumption and from Property 3.3 that the FT of $z(t)$ is zero outside the frequency interval $\nu_{0}<\nu<B$ and has a spectral line at the carrier frequency $\nu_{0}$. Consider now the signal $z^{*}(t)$. Its FT is equal to $Z^{*}(-\nu)$, and this FT is zero outside the frequency interval $-B<-\nu<-\nu_{0}$ and has a spectral line at the frequency $-\nu_{0}$. Let us now introduce the signal

$$
w(t)=z^{*}(t) \exp (2 \pi j f t)
$$

with $f>B$. This signal obviously satisfies $|w(t)|=1$, and the frequency condition ensures that it is an AS. It then has the general form (14) and (15) and must satisfy Property 3.3. However, its spectral line is at the frequency $f-\nu_{0}$, and $W(\nu)$ is not zero for $\nu<f-\nu_{0}$. This is in contradiction with Property 3.3, which shows the result.

Property 3.5-Frequency Shift: If in (14) we replace $\omega_{0}$ with $\omega_{1}$, with $\omega_{1}>\omega_{0}$, we obtain a complex signal that is still an AS. As a consequence, if $x(t)=\cos [\phi(t)]$ is a phase signal, which means that its phase has the structure (20), $x^{\prime}(t)=a \cos [\Delta \omega t+\phi(t)]$, where $\Delta \omega=\omega_{1}-\omega_{0}$, is still a phase signal with the carrier frequency $\omega_{1}$. This especially implies that $x^{\prime}(t)$ is a high-frequency $\left(\omega_{1}\right)$ signal.

Property 3.6-Instantaneous Frequency of a Phase Signal: It is obtained by differentiating the instantaneous phase. The most general form of this phase is given by (21) and (22), and differentiating this equation yields

$$
\omega(t)=\omega_{0}+2 \sum_{k=1}^{N} \frac{b_{k}}{b_{k}^{2}+\left(a_{k}-t\right)^{2}} .
$$

A similar equation has been obtained in [20] and [21] by using a rather different procedure.

As the coefficients $b_{k}$ are positive, because of the localization of the zeros $z_{k}$, we deduce that the instantaneous frequency $\omega(t)$ is always greater than $\omega_{0}$. This is another illustration of the fact that the FT of $x(t)$ is zero for $\nu<\omega_{0}$. If all the $z_{k}$ s are zero, then $b(t)$ defined by (15) is equal to one, and the instantaneous frequency is simply $\omega_{0}$.

Some other comments can be presented on the structure of the instantaneous frequency of a phase signal, and this is especially relevant in all those questions dealing with frequency or phase modulation of signals. The information carried by the instantaneous frequency of a phase signal is entirely in the term

$$
\omega_{m}(t)=2 \sum_{k=1}^{N} \frac{b_{k}}{b_{k}^{2}+\left(a_{k}-t\right)^{2}}
$$

where the index $m$ stands for the modulation term. We note that this function tends to zero when $|t| \rightarrow \infty$. This especially means that $\omega_{m}(t)$ cannot be a periodic function, and this is related to the fact that a phase signal cannot have spectral lines, except those coming from the carrier frequency $\omega_{0}$.

Furthermore, we note that $\omega_{m}(t)$ is a rational function in $t$. The polynomials appearing in the numerator and the 
denominator have the degrees $2 N-2$ and $2 N$, respectively. As $N$ is arbitrary, we deduce that by using the $2 N$ parameters $a_{k}$ and $b_{k}$, it is possible to approximate a large class of functions. The most limiting constraint on these functions comes from the necessary behavior for $|t| \rightarrow \infty$. In fact, $\omega_{m}(t)$ decreases at infinity in $|t|^{-2}$, which is a strong restriction on the instantaneous frequency.

\section{Unimodular Signals, Phase, AND FREQUENCY MODULATIONS}

\section{A. Definitions}

By definition, the instantaneous amplitude of a phase signal is constant. Consider now the function $w(t)=\exp [\phi(t)]$. Its modulus is one but, as, in general, $w(t)$ has no reason to be an AS, the instantaneous amplitude of $\cos [\phi(t)]$ has no reason to be constant. The signal $w(t)$ is said to be unimodular. The characteristic property of such a signal is, of course, $w(t) w^{*}(t)=1$, which especially implies that its energy is infinite. As a consequence, the calculation of its FT can require the use of distributions, and the best example of this point appears with the signal $w(t)=\exp \left[j 2 \pi \nu_{0} t\right]$. Its FT is $\delta\left(\nu-\nu_{0}\right)$, where $\delta(\cdot)$ is the Dirac distribution, and this introduces a spectral line at the frequency $\nu_{0}$. It results from the property $w(t) w^{*}(t)=1$ that the FT $W(\nu)$ of $w(t)$ satisfies

$$
\int W(n) W^{*}(n-\nu) d n=\delta(\nu)
$$

where $W(\nu)$ is the FT of $w(t)$. This means that the correlation function of $W(\nu)$ is a Dirac distribution.

We deduce from the previous discussion that a unimodular signal cannot be bandlimited except in the monochromatic case. Indeed, suppose that $W(\nu)$ is zero for $|\nu|>B$. Multiplying $w(t)$ by $\exp \left[j 2 \pi \nu_{0} t\right]$, where $\nu_{0}>B$ yields a signal that is both unimodular and analytic because its FT is zero for negative frequencies. It is then the AS of a phase signal whose FT is zero for $\nu>\nu_{0}+B$. This is in contradiction with Property 3.4.

It is worth pointing out that this does not mean that a signal such as $\cos [\phi(t)]$ cannot be bandlimited. Indeed, we have seen that the signal $\operatorname{sinc}(2 B t)$ can be written as $\cos [\phi(t)]$, and its FT is zero for $|\nu|>B$. However, this means that $\sin [\phi(t)]$ is not its Hilbert transform. Indeed, if this were true, $\cos [\phi(t)]$ would be a bandlimited phase signal, which is impossible.

For the following discussion, it is of interest to present some structures of the FT's of unimodular signals. There are unimodular signals with an FT containing only spectral lines. The best example appears when the function $\phi(t)$ is periodic with the period $T$. For instance, consider the signal $w(t)=$ $\exp j\left[\omega_{0} t+m \sin (\Omega t)\right]$. As $\exp j[m \sin (\Omega t)]$ is periodic with the period $T=2 \pi / \Omega$, its FT contains only spectral lines at the frequencies $n \Omega / 2 \pi$. By shifting these lines by $\omega_{0} / 2 \pi$, we obtain the FT of $w(t)$.

More generally, some unimodular signals can be written as

$$
w(t)=\exp [j \phi(t)]=\sum_{k} a_{k} \exp \left[j 2 \pi \nu_{k} t\right]
$$

and exhibit spectral lines at the frequencies $\nu_{k}$. It results from the unimodular property that the number of such spectral lines is either one or infinite. Indeed, the property $|w(t)|^{2}=1$ becomes

$$
\sum_{k} \sum_{k^{\prime}} a_{k} a_{k^{\prime}}^{*} \exp \left[j 2 \pi\left(\nu_{k}-\nu_{k^{\prime}}\right) t\right]=1
$$

for any $t$, and this is impossible if the number of frequencies $\nu_{k}$ is finite.

Some unimodular signals can also have an FT with only one spectral line and a continuous part. To present an example of such a situation, let $f(t)$ be the function equal to zero for $|t|>T / 2$ and to $\exp [j \phi(t)]-1$ otherwise. This function has a continuous FT $F(\nu)$, and this FT tends to zero when $|\nu|$ tends to infinity. Furthermore, the signal $w_{0}(t)=1+f(t)$ is unimodular because its value is 1 for $|t|>T / 2$ and $\exp [j \phi(t)]$ otherwise. Its FT is $W(\nu)=\delta(\nu)+F(\nu)$. Finally, the signal $w(t)=w_{0}(t) \exp \left[j\left(\omega_{0} t+\theta\right)\right]$ is unimodular, and its FT is $\delta\left(\nu-\nu_{0}\right)+F\left(\nu-\nu_{0}\right)$. This shows the presence of a spectral line at the frequency $\nu_{0}$ and a continuous part due to the function $F(\cdot)$.

Any signal with at least one spectral line necessarily has an infinite energy. Conversely, the energy can be infinite without a spectral line, and we shall verify that this situation can appear for unimodular signals. The energy of the signal $w(t)$ satisfying $w(t) w^{*}(t)=1$ is also the integral of $|W(\nu)|^{2}$. This integral can become infinite either if $|W(\nu)|^{2}$ is not bounded or if it decreases too slowly at infinity or for the two reasons simultaneously.

Consider, for example, the signal $w(t)=\operatorname{Sg}(t)=t /|t|$. It is obviously unimodular as well as real. Its FT is $1 / j \pi \nu$ and is not bounded for $\nu=0$. However, there is no spectral line at the frequency zero. As a consequence, the FT of the signal $w(t)=\operatorname{Sg}(t) \exp \left(j 2 \pi \nu_{0} t\right)$ is $1 / j \pi\left(\nu-\nu_{0}\right)$.

Let us now present an example of a unimodular signal with a bounded FT. Starting from the Fresnel integrals

$$
\int_{-\infty}^{+\infty} \cos \left(b x^{2}\right) d x=\int_{-\infty}^{+\infty} \sin \left(b x^{2}\right) d x=(\pi / 2 b)^{1 / 2}
$$

it is easy to show that the FT of the so-called complex chirp signal $w(t)=\exp \left(j b t^{2}\right)$ is

$$
W(\nu)=e^{j \pi / 4}(\pi / b)^{1 / 2} \exp \left[-j \pi^{2} b^{-1} \nu^{2}\right] .
$$

It is clear that $w(t)$ is a unimodular signal and that its FT is bounded because its modulus is constant and equal to $\pi / b$. In other words, the property of infinite energy comes from the fact that $w(t)$ and $W(\nu)$ have a constant modulus.

\section{B. Application in Phase and Frequency Modulation}

As stated in the introduction, there is a great tendency to consider that a signal $x(t)$ like $\cos [\phi(t)]$ is phase modulated, or to admit that $\phi(t)$ is its instantaneous phase, even if all the previous discussions show that this has no reason to be true. The complex signal $w(t)=\exp [j \phi(t)]$ is sometimes considered to be the complex representation of $x(t)$ (see [22, p. 13]) even if $w(t)$ has, in general, no reason to be an AS. It is then worth analyzing the difference between 
the unimodular signal $w(t)$ and the AS of $\cos [\phi(t)]$. This introduces the difference between $\phi(t)$ and the instantaneous phase, as defined previously, by using the AS.

In reality, this difference is very low in many practical cases. This is the reason why a good understanding of frequency modulation in radio engineering has been realized long before the AS was invented. In some sense, the practice was in advance with respect to the theory.

The major argument justifying the intuitive concept of instantaneous phase comes from an application of the Bedrosian theorem to situations where it cannot be strictly applied. This theorem indicates that if a signal $w(t)$ is bandlimited in the frequency domain $|\nu|<B$, the signal $w(t) \exp \left(j 2 \pi \nu_{0} t\right)$ is an AS as soon as $\nu_{0}>B$. Applied to a unimodular signal $w(t)=\exp [j \phi(t)]$, this means that if it is bandlimited, then $\exp j\left[2 \pi \nu_{0} t+\phi(t)\right]$ is an AS for $\nu_{0}$ sufficiently large, and $2 \pi \nu_{0} t+\phi(t)$ becomes the instantaneous phase of $\cos \left[2 \pi \nu_{0} t+\right.$ $\phi(t)]$. However if $\exp [j \phi(t)]$ is bandlimited, this is also the case of $\exp j\left[2 \pi \nu_{0} t+\phi(t)\right]$, and therefore, this signal is a unimodular and bandlimited AS. We have seen above that this is impossible. Then, there is a contradiction in this reasoning. However, this contradiction comes from the high frequencies of the spectrum, and there is a great tendency to consider that arbitrary high frequencies do not have any physical meaning. This tendency leads us to consider that all the real signals of radio engineering are practically bandlimited.

It is not possible to reconcile rigorously these theoretical and practical approaches. It is clear that any physical device cannot transmit arbitrary high frequencies. However, the consideration of the chirp signal introduced above indicates that its FT given by (31) does not decrease at infinity, and such frequencies are, therefore, present in its spectrum.

The only scientific procedure would require the calculation of the error coming from the approximations introduced in many practical considerations, and we shall now indicate some possible approaches of this question.

This problem is not new and has been approached in various ways. As indicated in [23], the difference between the AS of $\cos [\phi(t)]$ and $\exp [j \phi(t)]$ appears only in their imaginary part, and this difference comes from the fact that (9) is not necessarily valid. As a consequence, it is possible to characterize this difference by the error signal

$$
\epsilon(t)=H\{\cos [\phi(t)]\}-\sin [\phi(t)]
$$

where $H(\cdot)$ denotes the Hilbert transform. There is no general procedure to calculate this error signal, and the results depend on the structure of the function $\phi(t)$.

For example, if $\phi(t)=b t^{2}$, we obtain the real chirp signal $x(t)=\cos \left(b t^{2}\right)$. It is shown in the Appendix that its Hilbert transform is

$$
\begin{aligned}
H\left[\cos \left(b t^{2}\right)\right]=\sqrt{\frac{2}{\pi}}\left[[C(\sqrt{b} t)+S(\sqrt{b} t)] \sin \left(b t^{2}\right)\right. \\
\left.+[C(\sqrt{b} t)-S(\sqrt{b} t)] \cos \left(b t^{2}\right)\right]
\end{aligned}
$$

where the functions $C(x)$ and $S(x)$ are also defined in this Appendix. This equation exhibits an interesting result. When $t$ goes to infinity, the functions $C(x)$ and $S(x)$ tend to the same limit given by (30), and taking into account the value of this limit, one sees that $H\left[\cos \left(b t^{2}\right)\right]$ tends to $H\left[\sin \left(b t^{2}\right)\right]$. This means that for $t$ sufficiently large, and this depends, of course, of the value of the constant $b$, the complex chirp signal $\exp \left(j b t^{2}\right)$ tends to become an analytical signal. This result has nothing to do with a frequency band limitation because the spectrum of $\exp \left(j b t^{2}\right)$ deduced from (31) does not present any decreasing for high frequencies.

Another possible approach is to calculate (possibly by computation) the FT $W(\nu)$ of $w(t)$ and to study its value for negative frequencies. Indeed, it is the fact that $W(\nu) \neq 0$ for $\nu<0$ that implies that $w(t)$ is not an AS, and therefore, $\phi(t)$ is not the instantaneous phase. Unfortunately, there is no general result in this way, and the calculation must be processed for each case considered.

More recently, the class of asymptotic AS's was introduced [24]. The idea is to consider unimodular signals that are only asymptotically AS. However, the conditions that such signals must satisfy are not easy to verify.

We shall, however, present the concept of asymptotic AS in another way. As indicated previously, the question is to know in which sense $\exp [j \phi(t)]$ can be considered to be approximately bandlimited. For this discussion, it is worth considering separately the case of signals with only spectral lines or without such lines.

Starting from the spectral representation (28), we can calculate the corresponding correlation function defined by

$$
\gamma(\tau)=\lim _{T \rightarrow \infty} \frac{1}{T} \int_{-T / 2}^{T / 2} w(t) w^{*}(t-\tau) d t
$$

and we have, of course, $\gamma(0)=1$ because $|w(t)|^{2}=1$. By using (28), we easily deduce

$$
\gamma(0)=\sum\left|a_{k}\right|^{2} \exp \left(2 \pi j \nu_{k} \tau\right)
$$

which means that the coefficients $\left|a_{k}\right|^{2}$ are the those of the power spectrum of $w(t)$. As this power is 1 , there is certainly a frequency $B$ such that the sum $\sum\left|a_{k}\right|^{2}$ calculated for all the frequencies greater than $B$ is arbitrary small. This means that the difference between $w(t)$ and the bandlimited signal obtained by the previous procedure has an arbitrary small power.

\section{Properties of Canonical Pairs}

In the previous sections, we have investigated properties of amplitude modulated signals like (1) or of phase signals like (2). Combining these results, it is interesting to exhibit some properties of signals like (5) in which the pair $[a(t), \phi(t)]$ is obviously canonical, which means that $a(t) \exp [j \phi(t)]$ is an AS.

The first point to note is that the canonical character of $[a(t), \phi(t)]$ does not, in general, imply any frequency constraint on the amplitude $a(t)$, as is sometimes believed [25]. This is the case, as seen in Section II, when the phase is linear in time or, equivalently, when the instantaneous frequency is constant. However, there is no reason for that to remain true for a more general structure of the instantaneous phase. In order to confirm this point, let us now present an example of 
instantaneous amplitude without any spectral limitation. Let $z(t)$ be defined by $(\alpha-2 \pi j t)^{-2}, \alpha>0$. It is obviously an AS because of the localization of the pole of this rational function. Its instantaneous amplitude $|z(t)|$ is $\left(\alpha^{2}+4 \pi^{2} t^{2}\right)^{-1}$, and the FT of this function is $(2 \alpha)^{-1} \exp [-\alpha|\nu|]$, which is never zero.

However, some spectral properties can be sufficient conditions to ensure that a pair $[a(t), \phi(t)]$ is canonical. More precisely, if the FT of $a(t)$ is limited to the frequencies $\nu$ satisfying $-B<\nu<+B$ and if the FT of $w(t)=\exp [j \phi(t)]$ is zero for $\nu<B$, the pair $[a(t), \phi(t)]$ is canonical. Indeed, the FT of $z(t)=a(t) w(t)$ is

$$
Z(\nu)=\int A(n) W(\nu-n) d n .
$$

The frequency limitation implies that $-B<n<B$ and $\nu-n>B$. This is impossible for $\nu<0$, and then, $Z(\nu)=0$ for $\nu<0$.

Nevertheless, it is worth pointing out that the condition on $\phi(t)$ is very restrictive because it implies that $\exp [j \phi(t)]$ is an AS. As a consequence, $\phi(t)$ must have the form (20). Furthermore, this condition is a band limitation of $w(t)$ and not of $\cos [\phi(t)]$. It is perfectly possible that the FT of $\cos [\phi(t)]$ is zero for $|\nu|<B$ without having $W(\nu)=0$ for $\nu<B$. This can be shown by contradiction. Suppose, therefore, that the condition on $\cos [\phi(t)]$ implies that the FT $W(\nu)$ of $w(t)=\exp [j \phi(t)]$ is zero for $\nu<B$. This implies that $w(t)$ is an AS and, more precisely, the AS of $\cos [\phi(t)]$. Let us show by example that this is not necessarily true. Let us take once again the signal (11). It can obviously be written as $\cos [\phi(t)]$. However, its AS is $\operatorname{sinc}(2 B t) \exp \left[j \omega_{0} t\right]$, which is not a unimodular signal. As a consequence, the AS cannot be written $\exp [j \phi(t)]$.

It is also worth pointing out that if $z_{1}(t)$ and $z_{2}(t)$ are two AS's, then the product $z(t)=z_{1}(t) z_{2}(t)$ is also an AS. This comes from the fact that the FT of $z(t)$ is given by the convolution integral

$$
Z(\nu)=\int Z_{1}(n) Z_{2}(\nu-n) d n .
$$

However, $Z_{1}(\nu)$ and $Z_{2}(\nu)$ are zero for $\nu<0$. The limits of integration are then $0<n<\nu$, and this yields $Z(\nu)=0$ for $\nu<0$. A direct consequence of this result is that if $[a(t), \phi(t)]$ is a canonical pair, the same is valid for $\left[a(t), \phi^{\prime}(t)\right]$, with $\phi^{\prime}(t)=\theta+\omega t+\phi_{b}(t)$, where $\phi_{b}(t)$ is a Blaschke phase defined by (19). This result comes from the fact that $a(t) \exp [j \phi(t)]$ is, by construction, an AS, and $\exp [j \phi(t)]$ is also an AS, as seen in Section III.

Finally, it is possible to use the previous results by combining the properties of amplitude and phase signals. More precisely, if $\left[a(t), \omega_{0} t\right]$ and $\left[1, \omega_{0} t+\phi_{b}(t)\right]$ are two canonical pairs, the same is valid for $\left[a(t), \omega_{0} t+\phi_{b}(t)\right]$.

At the end of this section, the concept of minimum phase signals is worth indicating, especially that studied in [20]. It is well known that the real and imaginary parts of an AS are Hilbert transforms of each other. This means that it is possible to deduce the imaginary part from the real part. The same property is no longer true for the amplitude and the phase. This obviously appears just above because we have seen that it is possible to change the phase without changing the amplitude. This property is well known in the context of causality properties of linear filters. It is known that if a filter is causal, the magnitude of its frequency response does not determine its phase, except in the case of minimum-phase filters. It is possible to transpose all these properties to the case of signals and especially to change the instantaneous phase of signals by manipulations of zeros in the complex plane (see [21]). This does not directly concern the questions analyzed in this paper.

\section{CASE OF RANDOM Signals}

Because of the importance of randomness in signal processing and communication problems, it is worth extending the previous results and introducing the concepts of random amplitude and phase. Let $(\Lambda, F, P)$ be a probability space. A complex random signal can be written as $z(t ; \lambda)$, where $t$ refers to the time and $\lambda$ to the randomness. The definitions of instantaneous amplitude and phase given in Section II make use of the AS of a real signal $x(t)$. These definitions can be transposed to the random case because the AS of a real random signal $x(t ; \lambda)$ is a well-defined concept. It can be introduced either in the time domain by

$$
z(t ; \lambda)=x(t ; \lambda)+j H[x(t ; \lambda)]
$$

where $H[\cdot]$ means the Hilbert transform, or in the frequency domain. For this, it is necessary to assume that $x(t ; \lambda)$ is harmonizable (see [26, p. 200]) or can be written as

$$
x(t ; \lambda)=\int d X(\nu ; \lambda) \exp (2 \pi j \nu t) .
$$

The AS $z(t ; \lambda)$ is then defined by the same integral where $d X(\nu ; \lambda)$ is replaced by $d Z(\nu ; \lambda)=2 d X(\nu ; \lambda)$ for $\nu>0$ and $d Z(\nu ; \lambda)=0$ for $\nu<0$.

If $x(t ; \lambda)$ is second-order stationary (see [26, p. 178]), it has a power spectrum $\Gamma_{x}(\nu)$, and the power spectrum $\Gamma_{z}(\nu)$ of its AS is zero for $\nu<0$. Conversely, this property characterizes a second-order stationary AS.

Consider first a signal like (1), where $m(t)$ is now random and harmonizable with spectral increments $d M(\nu ; \lambda)$. We can transpose without alteration the results of Section II-B, and the consequence is that $m(t)$ is the instantaneous amplitude of (1) if and only if $m(t ; \lambda)$ is a low-frequency $\left(\omega_{0} / 2 \pi\right)$ signal. This means that the increments of its spectral representation satisfy $d M(\nu ; \lambda)=0$ for $\nu>\nu_{0}=\omega_{0} / 2 \pi$.

As in the nonrandom case, the question becomes more complicated for the condition on the instantaneous phase. For its study, consider first the cases of phase and unimodular random signals. A random phase signal $x(t ; \lambda)$ is such that for each value of $\lambda, x(t ; \lambda)$ is a deterministic phase signal. This means that each trajectory is a phase signal, as defined in Section III-A. It has been shown that such signals are defined by $2 N+2$ parameters appearing in (14) and (15). It suffices to assume that these parameters are random in order to introduce random phase signals.

Suppose first that in $(14), b(t)=1$. This means that there are only two possible random parameters: $\omega_{0}(\lambda)$ and $\theta(\lambda)$. In 
this case, $z(t)$ is second-order stationary, and its correlation function is

$$
\gamma_{z}(\tau) \triangleq E\left[z(t) z^{*}(t-\tau)\right]=E[\exp (2 \pi j \nu(\lambda) \tau)]
$$

where $\nu(\lambda)=\omega_{0}(\lambda) / 2 \pi$. This expression exhibits the characteristic function of the random variable $\nu(\lambda)$, and introducing its probability density function $p(f)$ yields

$$
\gamma_{z}(\tau)=\int \exp (2 \pi j f \tau) p(f) d f
$$

which shows that the power spectrum $\Gamma_{z}(\nu)$ of $z(t ; \lambda)$ is simply $p(\nu)$. As a consequence, $z(t ; \lambda)$ is an AS if and only if $p(\nu)=0$ for $\nu<0$, which means that the random frequency takes only positive values. This implies, indeed, that each trajectory is a real monochromatic signal of frequency $\nu$.

The impact of random Blaschke factor is much more difficult to analyze. In order to understand this point, suppose that in (15), $N=1$. This leads us to write $b(t)$ as

$$
b(t)=\frac{t-z}{t-z^{*}}=1-\frac{z-z^{*}}{t-z^{*}}
$$

and its FT is readily

$$
B(\nu)=\delta(\nu)-4 \pi b u(\nu) \exp (-2 \pi b \nu) \exp (-2 \pi j a \nu)
$$

where $u(\nu)$ is the unit step function, and $z=a+j b$ with $b>0$. We assume now that $a$ and $b$ are two real random variables $a(t ; \lambda)$ and $b(t ; \lambda)$. It is clear in (42) and (43) that $b(t ; \lambda)$ cannot be stationary, which is rather inconvenient for the description of many physical phenomena. However, any trajectory of $b(t ; \lambda)$ is an AS, which implies that $b(t ; \lambda)$ is also a random AS.

Let us now consider the case of unimodular complex signals. Following the previous discussion, we shall assume that there exists a carrier frequency $\omega_{0}$ such that the signal can be expressed as

$$
w(t)=\exp \left(j \omega_{0} t\right) \exp [j \Phi(t ; \lambda)]
$$

where $\Phi(t ; \lambda)$ is now a random function. We deduce from this equation that

$$
w(t) w^{*}(t-\tau)=\exp \left(j \omega_{0} \tau\right) \exp [j \Delta \Phi(t, \tau ; \lambda)]
$$

where $\Delta[\Phi(t, \tau ; \lambda)]=\Phi(t ; \lambda)-\Phi(t-\tau ; \lambda)$ is the increment of the random function $\Phi(t ; \lambda)$ between $t-\tau$ and $t$. It results from this relation that in order to obtain that $w(t)$ is secondorder stationary, we must assume that $\Phi(t ; \lambda)$ is a signal with stationary increments (see [26, p. 289]). Such signals can be expressed for $t>0$ as

$$
\Phi(t ; \lambda)=\int_{0}^{t} F(\theta ; \lambda) d \theta+\Phi_{0}(\lambda)
$$

where $F(\theta ; \lambda)$ is a stationary signal. As a consequence, the increment appearing in (45) takes the form

$$
\Delta \Phi(t, \tau ; \lambda)=\int_{t-\tau}^{t} F(\theta ; \lambda) d \theta .
$$

This increment is then obtained by filtering $F(t ; \lambda)$ using a linear filter with impulse response equal to one for $0<t<\tau$ and zero otherwise. As $F(t ; \lambda)$ is assumed to be stationary, the statistical properties of $\Delta \Phi(t, \tau ; \lambda)$ do not depend on $t$ but are functions of $\tau$ only.

The correlation function of $w(t)$ is deduced from (45) by

$$
\gamma_{z}(\tau)=\exp \left(j \omega_{0} \tau\right) E[\exp \{j \Delta \Phi(t, \tau ; \lambda)\}] .
$$

As in (40), the last term of this equation is the characteristic function of the random variable $\Delta \Phi(t, \tau ; \lambda)$ given by (47). As stated before, this characteristic function does not depend on $t$. However, its calculation from the statistical properties of $F(t ; \lambda)$ is, in general, impossible without any additional assumption on the function $F(t ; \lambda)$. The simplest possible, which also is the most important in physical applications, is to assume that $F(t ; \lambda)$ is Gaussian (or normal). In this case, any linear transformation preserves the Gaussian character, and as a consequence, $\Delta \Phi(t, \tau ; \lambda)$ is a Gaussian random variable. It is then entirely characterized by its mean value and its variance. We assume that the expectation is zero, which physically means that the mean frequency of $w(t)$ is $\omega_{0}$. By applying the classical formulae of linear filtering, we can express the variance of $\Delta \Phi(t, \tau ; \lambda)$ in terms of the power spectrum of $F(t)$ by

$$
\sigma_{\Delta \Phi}^{2}=\tau^{2} \int \operatorname{sinc}^{2}(\nu \tau) \Gamma_{F}(\nu) d \nu .
$$

Two extreme cases are of interest. The first appears when the function $F(t ; \lambda)$ does not depend on $t$. This yields that $\Phi(t)=F t$, and we come back to (40) with $F(\lambda)=2 \pi \nu(\lambda)$. The power spectrum $\Gamma_{F}(\nu)$ in (49) becomes $\sigma_{F}^{2} \delta(\nu)$, and the variance $\sigma_{\Delta \Phi}^{2}$ is then $\sigma_{F}^{2} \tau^{2}$. As a consequence, $\Delta \Phi(t, \tau ; \lambda)$ is a random variable with the distribution $N\left(0, \sigma_{F}^{2} \tau^{2}\right)$, and its characteristic function is $\exp \left[-(1 / 2) \sigma_{F}^{2} \tau^{2}\right]$ in such a way that (48) becomes

$$
\gamma_{z}(\tau)=\exp \left(j \omega_{0} \tau\right) \exp \left[-(1 / 2) \sigma_{F}^{2} \tau^{2}\right] .
$$

Its FT has still a normal shape centered at the frequency $\nu_{0}=\omega_{0} / 2 \pi$ and a standard deviation of $\sigma_{F}$. As a consequence, the FT of $\gamma_{z}(\tau)$, which still has a normal shape, is never zero, and $\gamma_{z}(\tau)$ is not an AS. However, it becomes practically an AS when the contribution of negative frequencies in the power spectrum can be neglected. This appears when $\sigma_{F} \ll \nu_{0}$. This condition means that the signal $x(t)=\cos \left[\omega_{0} t+\Phi(t ; \lambda)\right]$ is asymptotically a phase signal with the instantaneous phase $\Phi(t ; \lambda)$.

Let us now consider the opposite case where $F(t)$ is a white noise or has a flat spectrum with a spectral level $c$. The variance (49) then takes the form $\sigma_{\Delta \Phi}^{2}=c \tau$, and the correlation function (48) becomes

$$
\gamma_{z}(\tau)=\exp \left(j \omega_{0} \tau\right) \exp [-(1 / 2) c|\tau|] .
$$

The corresponding spectrum has a Lorentzian shape (see [26, p. 173]). However, regardles of this form, the previous condition remains true. If $c \ll \nu_{0}$, the unimodular signal (44) tends to become an AS of a real random phase signal.

Starting from these properties of phase and unimodular random signals, it is possible to extend without difficulty to the random case the results obtained in Sections IV-B and V for deterministic signals. 


\section{APPENDIX}

Let $C(x)$ and $S(x)$ be the functions defined by

$$
C(x)=\int_{0}^{x} \cos u^{2} d u, \quad S(x)=\int_{0}^{x} \sin u^{2} d u .
$$

By applying the Cauchy theorem for integration in the complex plane, we obtain $C(\infty)=S(\infty)=(\pi / 8)^{1 / 2}$, which gives the values of the Fresnel integrals (30). From these integrals, we deduce that

$$
\int_{-\infty}^{+\infty} \exp \left( \pm j b x^{2}\right) d x=(\pi / b)^{1 / 2} \exp ( \pm j \pi / 4) .
$$

Consider now the real chirp signal $\cos \left(b t^{2}\right)$. By using the previous equations, it is possible to calculate its FT, and the result is

$$
X(\nu)=\sqrt{\frac{\pi}{b}} \cos \left[\frac{\pi^{2} \nu^{2}}{b}-\frac{\pi}{4}\right] .
$$

Its AS is defined from its FT equal to 0 for $\nu<0$ and to $2 X(\nu)$ for $\nu>0$. After some algebra, one obtains the imaginary part of this AS, or the Hilbert transform of $\cos \left(b t^{2}\right)$, which is given by (33).

\section{ACKNOWLEDGMENT}

The author would like to acknowledge P. Flandrin from École Normale Supérieure de Lyon and Y. Tanguy from École Supérieure d'Électricité for many helpful discussions on phase signals.

\section{REFERENCES}

[1] P. Panter, Modulation, Noise and Spectral Analysis. New York: McGraw-Hill, 1965.

[2] J. M. Wozencraft and I. M. Jacobs, Principles of Communication Engineering. New York: Wiley, 1965.

[3] A. V. Oppenheim and A. S. Willsky, Signals and Systems. Englewood Cliffs, NJ: Prentice-Hall, 1983.

[4] M. Schwartz, Information Transmission Modulation and Noise. New York: McGraw-Hill, 1987.

[5] H. Taub and D. L. Schilling, Principles of Communication Systems. New York: McGraw-Hill, 1986

[6] B. Picinbono, Principles of Signals and Systems. London, U.K.: Artech House, 1988

[7] B. Boashash, "Estimating and interpreting the instantaneous frequency of a signal," Proc. IEEE, vol. 80, pp. 520-538, 1992.

[8] D. Gabor, "Theory of communications," J. Inst. Elec. Eng., vol. 93, pp. 429-457, 1946.

[9] J. Ville, "Théorie et applications de la notion de signal analytique," Cables et Transmissions, vol. 2, pp. 61-74, 1948.
[10] J. Oswald, "The theory of analytic band-limited signals applied to carrier systems," IRE Trans. Commun. Theory, vol. CT-3, pp. 244-251, 1956.

[11] J. Dugundji, "Envelopes and preenvelopes of real waveforms," IRE Trans. Inform. Theory, vol. 4, pp. 53-57, 1958.

[12] E. Bedrosian, "The analytic signal representation of modulated waveforms," Proc. IRE, vol. 50, pp. 2071-2076, 1962.

[13] D. Vakman, "On the definition of concepts of amplitude, phase and instantaneous frequency of a signal," Radio Eng. Electron. Phys., pp. 754-759, 1972.

[14] _ "On the analytic signal, the Teager-Kaiser energy algorithm, and other methods for defining amplitude and frequency," IEEE Trans. Signal Processing, vol. 44, pp. 791-797, Apr. 1996.

[15] P. Loughlin and B. Tacer, "On the amplitude and frequency-modulation decomposition of signals," J. Acoust. Soc. Amer., vol. 100, pp. 1594-1601, Sept. 1996.

[16] E. Bedrosian, "A product theorem for Hilbert transforms," Proc. IEEE, vol. 51, pp. 868-869, 1963

[17] B. Picinbono, "Représentation des signaux par amplitude et phase instantanées," Ann. Télécommun., vol. 38, pp. 179-190, 1983.

[18] S. F. Edwards and G. B. Parrent, "The form of the general unimodular analytic signal," Optica Acta, vol. 6, pp. 367-371, 1959.

[19] R. Nevanlinna, Analytic Functions. Berlin: Springer-Verlag, 1970.

[20] H. Voelcker, "Toward a unified theory of modulation. Part 1, Phase envelopes relationships," Proc. IEEE, vol. 54, pp. 340-353, 1966.

[21] H. Voelcker, "Toward a unified theory of modulation. Part 2, Zero manipulation," in Proc. IEEE, vol. 54, pp. 737-755, 1966.

[22] G. Brun, Modulations Analogiques. Paris: Eyrolles, 1991.

[23] W. L. Rubin and J. V. Di Franco, "Analytic representation of wide-band radio frequency signals,” J. Franklin Inst., vol. 275, pp. 197-204, 1966.

[24] N. Delprat et al., "Asymptotic wavelets and Gabor analysis; extraction of instantaneous frequencies," IEEE Trans. Inform. Theory, vol. 38, pp. 644-664, 1992.

[25] R. Gendrin and P. Robert "Temps de groupe et largeur de bande de signaux modulés simultanément en amplitude et en fréquence," Ann. Télécommun., vol. 37, pp. 289-297, 1982.

[26] B. Picinbono, Random Signals and Systems. Englewood Cliffs, NJ: Prentice-Hall, 1993.

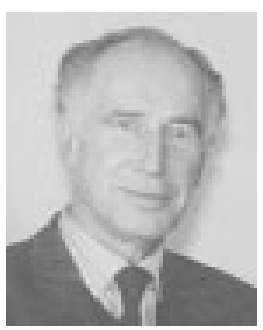

Bernard Picinbono (M'63-SM'79-F' 85) was born in Algiers, Algeria, on March 21, 1933. He received the Agrégation des Sciences Physiques degree in 1956 and the Doctorat d'état degree in 1960.

From 1960 to 1965, he was a Professor at the University of Algiers, Algeria, and since 1965, he has been with the Department of Physics of the University of Paris-Sud, Orsay, France. He was the President of this university from 1970 to 1975 . He was Director of the Laboratoire des Signaux et Systèmes, a joint laboratory of the Centre National de la Recherche Scientifique (CNRS), the École Supérieure d'Électricité (Supélec) and the University of Paris-Sud between 1975 and 1987. From 1990 to 1995, he was General Director of Supélec. His research interests include statistical signal processing, detection and estimation, and stochastic processes. He is the author of numerous papers and books on these subjects.

Dr. Picinbono is a member of the French Academy of Sciences and President of the GRETSI, with which he organizes, every two years in France, a conference that is mainly devoted to signal and image processing. 